

\title{
On the evaluation of 'saving-consumption' plans
}

\author{
Steven Vanduffel Jan Dhaene Marc Goovaerts
}

July 13, 2004

\begin{abstract}
Knowledge of the distribution function of the stochastically compounded value of a series of future (positive and/or negative) payments is needed for solving several problems in an insurance or finance environment, see e.g. Dhaene et al. (2002 a,b). In Kaas et al. (2000), convex lower bound approximations for such a sum have been proposed. In case of changing signs of the payments however, the distribution function or the quantiles of the lower bound are not easy to determine, as the approximation for the random compounded value of the payments will in general not be a comonotonic sum.

In this paper, we present a method for determining accurate and easy computable approximations for risk measures of such a sum, in case one first has positive payments (savings), followed by negative ones (consumptions).

This particular cashflow pattern is observed in 'saving - consumption' plans. In such a plan, a person saves money on a regular basis for a certain number of years. The amount available at the end of this period is then used to generate a yearly pension for a fixed number of years. Using the results of this paper one can find accurate and easy to compute answers to questions such as: "What is the minimal required yearly savings effort $\alpha$ during a fixed number of years, such that one will be able to meet, with a probability of at least $(1-\varepsilon)$, a given consumption pattern during the withdrawal period ?"
\end{abstract}




\section{Introduction}

In a finance or insurance context, one is often interested in the distribution function (d.f.) of a random variable (r.v.) $S$ given by

$$
S=\sum_{i=0}^{n} \alpha_{i} e^{Z_{i}} .
$$

Here the $\alpha_{i}$ are real numbers and $\left(Z_{0}, Z_{1}, \ldots, Z_{n}\right)$ is a multivariate normal random vector.

The accumulated value at time $n$ of a series of future deterministic saving amounts $\alpha_{i}$ at times $i, i=0, \ldots, n-1$, can be written in the form (1), where $Z_{i}$ denotes the random accumulation factor over the period $[i, n]$.

The present value of a series of future deterministic payments $\alpha_{i}$ at times $i, i=1, \ldots, n$, can be written in the form (1), where now $Z_{i}$ denotes the random discount factor over the period $[0, i]$.

Determining the price of Asian or basket options in a Black \& Scholes setting boils down to computing stop-loss premiums of a r.v. of the type described in (1), see for instance Albrecher, Dhaene, Goovaerts \& Schoutens (2003) or Vanmaele, Dhaene, Deelstra, Liinev \& Goovaerts (2004).

As mentioned in Dhaene, Vanduffel, Goovaerts, Kaas \& Vyncke (2004) or Vanduffel, Dhaene, Goovaerts \& Kaas. (2003), setting provisions and capital requirements in an insurance context comes down to determining risk measures related to a r.v. of the type (1).

As the r.v. $S$ defined in (1) is a sum of non-independent lognormal r.v.'s, its d.f. cannot be determined analytically. Therefore a variety of approximation techniques for determining d.f.'s of this type have been proposed in the literature. Practitioners often use a (first two moments) matching lognormal approximation for the d.f. of $S$. Milevsky \& Posner (1998) and Milevsky \& Robinson (2000) propose a moment matching reciprocal Gamma approximation for the d.f. of $S$.

Kaas, Dhaene and Goovaerts (2000) and Dhaene, Denuit, Goovaerts, Kaas \& Vyncke (2002a,b) derive a lower bound approximation for the d.f. of $S$. The lower bound is defined by $\mathrm{E}[S \mid \Lambda]$ for an appropriate choice of the conditioning r.v. $\Lambda$. It is called a lower bound approximation since (the d.f.) of $\mathrm{E}[S \mid \Lambda]$ is smaller in the convex order sense than (the d.f. of) $S$. This means that the expectations of both r.v.'s are equal, whereas the stop-loss premiums of the lower bound are smaller than the corresponding stop-loss 
premiums of $S$. The lower bound approximation performs very accurate when an 'optimal' choice is made for the conditioning r.v. $\Lambda$, see for instance Vanduffel, Hoedemakers \& Dhaene (2004).

We point out that the lognormal and the reciprocal Gamma approximation have been proposed in the context of series of positive cash flows, whereas the lower bound approximation can also be applied in case of changing signs of the $\alpha_{i}$. In this case however, and this in contrast to the situation that all $\alpha_{i}$ have equal signs, it is not possible to find a conditioning r.v. $\Lambda$ that leads to an accurate approximation for the d.f. of $S$ such that $\mathrm{E}[S \mid \Lambda]=\sum_{i=0}^{n} \alpha_{i} \mathrm{E}\left[e^{Z_{i}} \mid \Lambda\right]$ is a sum of non-decreasing functions of $\Lambda$. This implies that distortion risk measures related to $\mathrm{E}[S \mid \Lambda]$ cannot be obtained by simply summing the corresponding risk measures of the individual terms in the sum, as is the case when all $\alpha_{i}$ are positive, see Dhaene, Vanduffel, Tang, Goovaerts, Kaas \& Vyncke (2004).

Goovaerts, De Schepper, Hua, Darkiewicz \& Vyncke (2003) propose an approach that uses convex bounds for the positive and negative sum separately. They connect the two r.v.'s involved by a copula of a particular family.

In this paper, we follow another path to determine accurate and easy computable approximations for a sum $S$ as defined in (1), in the special case that one first has positive payments $\alpha_{i}$ (savings) followed by negative ones (withdrawals).

An important situation where one encounters this particular cash flow pattern, and hence where our results can be applied, is the saving - consumption problem. Take as an example a 20/65/95 pension plan in a defined contribution pension scheme. A person of age 20 intends to save money for 45 consecutive years (until retirement). After his retirement, he wants to withdraw money from his pension account on a regular basis and this for a period of 30 years. Assume that his yearly savings are constant and equal to $\alpha$, while his yearly consumption(pension) is constant and equal to 1 . A relevant question to answer is: "What is the minimal required yearly savings effort $\alpha$ such that this person will be able to meet his consumption pattern during the 30 year withdrawal period, with a probability of at least $(1-\varepsilon)$ ? In this paper, we will present a methodology for answering these types of questions.

The paper is organized as follows. In Section 2, we describe the savingconsumption problem. In Sections 3 and 4, we present accurate and easy computable approximations for the quantiles of final wealth random variables in the saving-consumption problem. The special case of constant savings and 
constant withdrawals is considered in Section 5. Finally, in Section 6 the theoretical results presented in this paper are illustrated by some numerical examples.

\section{Problem description}

Consider a set of deterministic amounts $\alpha_{0}, \alpha_{1}, \cdots, \alpha_{n+m}$ with $n \geq 1, m \geq$ 0 . The first $n$ amounts $\alpha_{0}, \alpha_{2}, \cdots, \alpha_{n-1}$ are nonnegative and correspond to saving amounts that are put on an investment account at respective times $0,1, \cdots, n-1$. The last $m+1$ amounts $\alpha_{n}, \alpha_{n+1}, \cdots, \alpha_{n+m}$ are nonpositive and correspond to withdrawals from the account at times $n, n+1, \cdots, n+m$, respectively. We will assume that $\alpha_{0}>0$ and $\alpha_{n}<0$. We will call a plan as described above a saving - consumption plan.

We assume that the return on the account is generated by a geometric Brownian motion process. An amount of 1 available on the account at time $i-1$, is assumed to grow to the random amount $e^{Y_{i}}$ at time $i$. Hence, the r.v. $Y_{i}$ is the random return over the year $[i-1, i]$. The r.v.'s $Y_{i}$ are i.i.d. and normal distributed, with parameters $\mu-\frac{\sigma^{2}}{2}$ and $\sigma^{2}$.

Let $V_{j}$ denote the surplus at time $j$. By convention, the surplus at time $j$ has to be understood as the surplus just after saving or withdrawal. Starting from the initial value $V_{0}=\alpha_{0}$, the surplus $V_{j}$ available at time $j$ is given by the following recursive relation:

$$
V_{j}=V_{j-1} e^{Y_{j}}+\alpha_{j}, \quad j=1, \cdots, n+m .
$$

For the moment, we allow the surplus to become negative, which means that shortselling of units of the investment account is allowed. Note that for the 'first saving - later consuming' cash flow pattern as described in (2), we have that once the surplus becomes negative, it will stay negative over the whole remaining time period and no recovery is possible anymore. This ruin scenario can only occur from time $n$ (retirement) on.

Solving the recursion (2), we find that the final surplus $V_{n+m}$ can be written as

$$
V_{n+m}=\sum_{i=0}^{n+m} \alpha_{i} e^{z_{i}}
$$


where the r.v.'s $Z_{i}$ are given by

$$
Z_{i}=\sum_{j=i+1}^{n+m} Y_{j}, \quad i=0, \cdots, n+m,
$$

and where, by convention, $\sum_{j=r}^{s} a_{j}=0$ if $r>s$. The r.v. $Z_{i}$ is the accumulation factor over the period $[i, n]$. Its mean and variance are given by

$$
\mathrm{E}\left[Z_{i}\right]=(n+m-i)\left(\mu-\frac{\sigma^{2}}{2}\right)
$$

and

$$
\sigma_{Z_{i}}^{2}=(n+m-i) \sigma^{2} .
$$

As it is impossible to determine the d.f. of $V_{n+m}$ analytically, we propose to approximate it by the d.f. of

$$
V_{n+m}^{l}=\mathrm{E}\left[V_{n+m} \mid \Lambda\right]
$$

for some convenient choice of the conditioning r.v. $\Lambda$. We have that (the d.f. of) $V_{n+m}^{l}$ is a lower bound in the sense of convex order for (the d.f. of) $V_{n+m}$, see for instance Kaas, Dhaene \& Goovaerts (2000). In particular, we have that

$$
\mathrm{E}\left[V_{n+m}\right]=\mathrm{E}\left[V_{n+m}^{l}\right]=\sum_{i=0}^{n+m} \alpha_{i} e^{(n+m-i) \mu} .
$$

In the sequel, we will consider a conditioning r.v. $\Lambda$ which is a linear combination of the compounded returns $Z_{i}$ :

$$
\Lambda=\sum_{j=0}^{n+m} \gamma_{j} Z_{j}
$$

for suitable choices of the parameters $\gamma_{j}$. This r.v. can also be written in terms of the yearly returns:

$$
\Lambda=\sum_{j=1}^{n+m} \beta_{j} Y_{j}
$$

where the relation between the $\beta_{j}$ and the $\gamma_{j}$ is given by

$$
\beta_{j}=\sum_{k=0}^{j-1} \gamma_{k}
$$


For more details about these kind of approximations, its relation with the concept of comonotonicity and its applications in insurance and finance, see Dhaene, Denuit, Goovaerts, Kaas \& Vyncke (2002 a,b).

Let $U$ be a uniform $(0,1)$ r.v. and let $\Phi$ denote the standard normal d.f. After some straightforward derivations we find that the r.v. $V_{n+m}^{l}$ defined in (7) with $\Lambda$ given by (9) is distributed as

$$
V_{n+m}^{l} \stackrel{d}{=} \sum_{i=0}^{n+m} \alpha_{i} e^{\mathrm{E}\left[Z_{i}\right]+\frac{1}{2}\left(1-r_{i}^{2}\right) \sigma_{Z_{i}}^{2}+r_{i} \sigma_{Z_{i}} \Phi^{-1}(U)}
$$

where $\stackrel{d}{=}$ stands for 'equality in distribution' and where the coefficients $r_{i}$ are given by

$$
r_{i}=\frac{\operatorname{cov}\left(Z_{i}, \Lambda\right)}{\sigma_{Z_{i}} \sigma_{\Lambda}}=\frac{\sum_{j=i+1}^{n+m} \beta_{j}}{\sqrt{n+m-i} \sqrt{\sum_{j=1}^{n+m} \beta_{j}^{2}}}, \quad i=0, \cdots, n+m-1
$$

and

$$
r_{n+m}=0 \text {. }
$$

Notice that the last term in (12), and also in (3), reduces to the constant number $\alpha_{n+m}$.

For any real-valued r.v. $X$, we will denote its distribution function $\operatorname{Pr}[X \leq x]$ by $F_{X}(x)$. by

The lower quantiles $Q[X]$ and the upper quantiles $Q_{p}^{+}[X]$ of $X$ are defined

$$
\begin{aligned}
Q_{p}[X] & =\inf \left\{x \in R \mid F_{X}(x) \geq p\right\}, \\
Q_{p}^{+}[X] & =\sup \left\{x \in R \mid F_{X}(x) \leq p\right\}, \quad p \in(0,1),
\end{aligned}
$$

where by convention, $\inf \phi=+\infty$ and $\sup \phi=-\infty$. When $F_{X}$ is strictly increasing, we have that $Q_{p}[X]=Q_{p}^{+}[X]$.

Let $X$ and $g(X)$ be real-valued r.v.'s. If $g$ is non-decreasing and continuous, then

$$
\begin{aligned}
Q_{p}[g(X)] & =g\left(Q_{p}[X]\right), \\
Q_{p}^{+}[g(X)] & =g\left(Q_{p}^{+}[X]\right), \quad p \in(0,1) .
\end{aligned}
$$

Furthermore, for any real number $x$ number, we have

$$
\begin{aligned}
p & \leq F_{X}(x) \Longleftrightarrow Q_{p}[X] \leq x \\
\operatorname{Pr}[X<x] & \leq p \Longleftrightarrow x \leq Q_{p}^{+}[X], \quad p \in(0,1) .
\end{aligned}
$$


If all the terms $\alpha_{i} e^{\mathrm{E}\left[Z_{i}\right]+\frac{1}{2}\left(1-r_{i}^{2}\right) \sigma_{Z_{i}}^{2}+r_{i} \sigma_{Z_{i}} \Phi^{-1}(U)}$ in (12) are non-decreasing functions of $U$ (or all are non-increasing functions of $U$ ), then we say that $V_{n+m}^{l}$ is a comonotonic sum. In case all terms are non-decreasing, then also the continuous function $f$ defined by

$$
f(p)=\sum_{i=0}^{n+m} a_{i} e^{\mathrm{E}\left[Z_{i}\right]+\frac{1}{2}\left(1-r_{i}^{2}\right) \sigma_{Z_{i}}^{2}+r_{i} \sigma_{Z_{i}} \Phi^{-1}(p)}, \quad p \in(0,1),
$$

is non-decreasing. From (16) we find that in this case, the $p$-quantile of $V_{n+m}^{l}$ is given by

$$
Q_{p}^{+}\left[V_{n+m}^{l}\right]=f(p), \quad p \in(0,1) .
$$

Moreover, in case $V_{n+m}^{l}$ is a comonotonic sum, any distortion risk measure (such as $\mathrm{VaR}_{p}$ and $\mathrm{TVaR}_{p}$ ) related to $V_{n+m}^{l}$ equals the sum of the risk measures related to the marginal terms in (12), see for instance Dhaene, Vanduffel, Tang, Goovaerts, Kaas \& Vyncke (2004).

A conditioning r.v. $\Lambda$ that makes $f$ non-decreasing (and continuous) can always be found. Indeed, if we take all $\beta_{j} \geq 0$ for $j=1,2, \ldots n$, whereas $\beta_{j}=0$ for $j=n+1, n+2, \ldots, n+m$, then one has that $f$ is non-decreasing, so that (19) holds in this case. Of course, we cannot expect that such a choice of the parameters $\beta_{j}$ will in general lead to an accurate approximation for the d.f. of $V_{n+m}$.

It is clear that for appropriate choices of $\Lambda$, the r.v. $V_{n+m}^{l}$ will not necessarily be a comonotonic sum of lognormal r.v.'s. Hence, the function $f(p)$ will not be non-decreasing on the whole interval $(0,1)$. This implies that the quantiles of $V_{n+m}^{l}$ cannot be determined easily in this case because (16) can not be applied.

As noticed above, the cashflow pattern that we consider (first saving later consuming) implies that once the value $V_{j}$ become negative, no recovery is possible in the sense that all future values $V_{k}, k \geq j$, will be negative too.

From now on, we will assume that shortselling is not allowed. Hence, once the surplus reaches level 0 , no further withdrawals from the pension account are allowed. One can easily verify that under this assumption, the wealth $W_{k}$ available on the account at time $k$ can be expressed as follows in terms of the surplus $V_{k}$, defined in (2):

$$
W_{k}=\max \left[V_{k}, 0\right], \quad k=0, \cdots, n+m .
$$

Quantities helping to decide whether or not to underwrite the 'saving - consumption' plan are the quantiles and the distribution function of final wealth 
$W_{n+m}$. As we have that

$$
Q_{p}^{+}\left[W_{n+m}\right]=\sup \left\{x \mid \operatorname{Pr}\left[W_{n+m}>x\right] \geq 1-p\right\},
$$

the quantile $Q_{p}^{+}\left[W_{n+m}\right]$ can be interpreted as the largest amount of money that will be left at time $n+m$, with a probability of at least $(1-p)$.

A possible requirement for a 'saving - consumption' plan to be considered as feasible could be $Q_{0.05}^{+}\left[W_{n+m}\right]>0$. For a plan fulfilling this condition, there is a probability of (at least) $95 \%$ that one will be able to meet the desired consumption pattern, hence that there will be no consumption shortfall. For a given plan, one could be interested in the probability that no consumption shortfall will occur. This probability is given by $\operatorname{Pr}\left[W_{n+m}>0\right]$.

In general, the probabilities $\operatorname{Pr}\left[W_{n+m}>x\right]$ and the quantiles $Q_{p}^{+}\left[W_{n+m}\right]$ cannot be determined analytically. Therefore, we propose to approximate (the d.f. of ) $W_{n+m}$ by (the d.f. of) $W_{n+m}^{l}=\max \left[V_{n+m}^{l}, 0\right]$. From (12), we find that

$$
W_{n+m}^{l} \stackrel{d}{=} \max [f(U), 0]
$$

where the function $f$ is defined by (18).

We propose to approximate the tail probabilities $\operatorname{Pr}\left[W_{n+m}>x\right]$ and the quantiles $Q_{p}^{+}\left[W_{n+m}\right]$ by $\operatorname{Pr}\left[W_{n+m}^{l}>x\right]$ and $Q_{p}^{+}\left[W_{n+m}^{l}\right]$, respectively.

In the next section we will prove that under rather weak conditions the function $\max [f(p), 0]$ is non-decreasing and continuous. From (16) we find a straightforward way to compute the quantiles of $W_{n+m}^{l}$ in this case.

\section{Approximations for the quantiles of $W_{n+m}$}

The following lemma gives sufficient conditions for the function $\max [f(p), 0]$ to be non-decreasing.

Lemma 1 If all $\beta_{j}>0$, for $j=1,2, \ldots n+m$, then for all $p$ in the unit interval $(0,1)$, one has that $f(p) \geq 0$ implies $f^{\prime}(p)>0$.

Proof. From (13), we find that

$$
r_{i} \sigma_{Z_{i}}=\sigma \frac{\sum_{j=i+1}^{n+m} \beta_{j}}{\sqrt{\sum_{j=1}^{n+m} \beta_{j}^{2}}}, \quad i=0, \cdots, n+m-1 .
$$


Hence, all correlations $r_{i}>0$ for $i=0,1, \ldots n+m-1$ and the sequence $\left\{r_{i} \sigma_{Z_{i}}\right\}_{0<r<n+m}$ is strictly decreasing and strictly positive.

By application of the chain rule, we find for $p \in(0,1)$ that

$$
f^{\prime}(p)=\frac{1}{\Phi^{\prime}\left[\Phi^{-1}(p)\right]} \sum_{i=0}^{n+m-1} a_{i} e^{\mathrm{E}\left[Z_{i}\right]+\frac{1}{2}\left(1-r_{i}^{2}\right) \sigma_{Z_{i}}^{2}+r_{i} \sigma_{Z_{i}} \Phi^{-1}(p)} r_{i} \sigma_{Z_{i}} .
$$

Now assume that $f(p) \geq 0$ for some value of $p$ in the unit interval $(0,1)$. When $n+m=1$, it is straightforward to prove that $f^{\prime}(p)>0$.

Let us now assume that $n+m>1$. Since $\frac{1}{\Phi^{\prime}\left[\Phi^{-1}(p)\right]}>0$ and $\alpha_{n+m} \leq 0$, we find that

$$
\begin{aligned}
f^{\prime}(p) & >\frac{r_{n-1} \sigma_{Z_{n-1}}}{\Phi^{\prime}\left[\Phi^{-1}(p)\right]} \sum_{i=0}^{n+m-1} a_{i} e^{\mathrm{E}\left[Z_{i}\right]+\frac{1}{2}\left(1-r_{i}^{2}\right) \sigma_{Z_{i}}^{2}+r_{i} \sigma_{Z_{i}} \Phi^{-1}(p)} \\
& =\frac{r_{n-1} \sigma_{Z_{n-1}}}{\Phi^{\prime}\left[\Phi^{-1}(p)\right]}\left(f(p)-\alpha_{n+m}\right) \geq 0
\end{aligned}
$$

which ends the proof.

Notice that

$$
\sigma^{2} \beta_{j}=\operatorname{Cov}\left(Y_{j}, \Lambda\right), \quad j=1, \cdots, n+m .
$$

Hence, the condition that all $\beta_{j}>0$ in Lemma 1 means that any yearly return $Y_{j}$ is strictly positive correlated with the conditioning random variable $\Lambda$.

One can easily prove that when all $\beta_{j}>0$, one has that

$$
\lim _{p \rightarrow 0} f(p)=a_{n+m} \leq 0
$$

and

$$
\lim _{p \rightarrow 1} f(p)=+\infty
$$

When all $\beta_{j}$ are assumed to be strictly positive, we find from Lemma 1 that the function $\max [f(p), 0]$ is non-decreasing (and continuous) on the interval $(0,1)$. From (22) and (16) we see that the quantiles of $W_{n+m}^{l}$ can easily be determined analytically in this case:

$$
Q_{p}\left[W_{n+m}^{l}\right]=Q_{p}^{+}\left[W_{n+m}^{l}\right]=\max [f(p), 0], \quad p \in(0,1) .
$$


Under the conditions of Lemma 1, we find that the d.f. of $W_{n+m}^{l}$ can be determined from

$$
f\left(F_{W_{n+m}^{l}}(x)\right)=x, \quad x \geq 0,
$$

Indeed, we have that for any $x \geq 0$,

$$
\begin{aligned}
F_{W_{n+m}^{l}}(x) & =\sup \left\{p \epsilon(0,1) \mid p \leq F_{W_{n+m}^{l}}(x)\right\} \\
& =\sup \left\{p \epsilon(0,1) \mid Q_{p}\left[W_{n+m}^{l}\right] \leq x\right\} \\
& =\sup \{p \epsilon(0,1) \mid \max [f(p), 0] \leq x\} \\
& =\sup \{p \epsilon(0,1) \mid f(p) \leq x\}
\end{aligned}
$$

so that the relation (27) follows from (24), (25), Lemma 1 and the fact that $f$ is continuous on $(0,1)$.

\section{On the choice of the conditioning r.v. $\Lambda$}

In order to determine the optimal values for the coefficients $\beta_{j}$, we follow the procedure as explained in Vanduffel, Hoedemakers \& Dhaene (2004). They consider the case were all cash flow payments $\alpha_{i}$ are positive. But the procedure can easily be extended to the case of general $\alpha_{i}$.

We have that $\operatorname{Var}\left[V_{n+m}\right]$ and $\operatorname{Var}\left[V_{n+m}^{l}\right]$ are given by

$$
\operatorname{Var}\left[V_{n+m}\right]=\sum_{i=0}^{n+m} \sum_{j=0}^{n+m} \alpha_{i} \alpha_{j} e^{\mathrm{E}\left[Z_{i}\right]+\mathrm{E}\left[Z_{j}\right]+\frac{1}{2}\left(\sigma_{Z_{i}}^{2}+\sigma_{Z_{j}}^{2}\right)}\left(e^{\operatorname{Cov}\left(Z_{i}, Z_{j}\right)}-1\right)
$$

and

$$
\operatorname{Var}\left[V_{n+m}^{l}\right]=\sum_{i=0}^{n+m} \sum_{j=0}^{n+m} \alpha_{i} \alpha_{j} e^{\mathrm{E}\left[Z_{i}\right]+\mathrm{E}\left[Z_{j}\right]+\frac{1}{2}\left(\sigma_{Z_{i}}^{2}+\sigma_{Z j}^{2}\right)}\left(e^{r_{i} r_{j} \sigma_{Z_{i}} \sigma_{Z_{j}}}-1\right)
$$


respectively. Consider the following first order approximation for $\operatorname{Var}\left[V_{n+m}^{l}\right]$ :

$$
\begin{aligned}
\operatorname{Var}\left[V_{n+m}^{l}\right] & \approx \sum_{i=0}^{n+m} \sum_{j=0}^{n+m} \alpha_{i} \alpha_{j} e^{\mathrm{E}\left[Z_{i}\right]+\mathrm{E}\left[Z_{j}\right]+\frac{1}{2}\left(\sigma_{Z_{i}}^{2}+\sigma_{Z_{j}}^{2}\right)}\left(r_{i} r_{j} \sigma_{Z_{i}} \sigma_{Z_{j}}\right) \\
& =\sum_{i=0}^{n+m} \sum_{j=0}^{n+m} \alpha_{i} \alpha_{j} e^{\mathrm{E}\left[Z_{i}\right]+\mathrm{E}\left[Z_{j}\right]+\frac{1}{2}\left(\sigma_{Z_{i}}^{2}+\sigma_{Z_{j}}^{2}\right)}\left(\frac{\operatorname{Cov}\left[Z_{i}, \Lambda\right] \operatorname{Cov}\left[Z_{j}, \Lambda\right]}{\operatorname{Var}(\Lambda)}\right) \\
& =\frac{\left(\operatorname{Cov}\left(\sum_{i=0}^{n+m} \alpha_{i} e^{\mathrm{E}\left[Z_{i}\right]+\frac{1}{2} \sigma_{Z_{i}}^{2}} Z_{i}, \Lambda\right)\right)^{2}}{\operatorname{Var}(\Lambda)} \\
& =\left(\operatorname{Corr}\left(\sum_{j=0}^{n+m} \alpha_{j} e^{\mathrm{E}\left[Z_{j}\right]+\frac{1}{2} \sigma_{Z_{j}}^{2}} Z_{j}, \Lambda\right)\right)^{2} \operatorname{Var}\left(\sum_{j=0}^{n+m} \alpha_{j} e^{\mathrm{E}\left[Z_{j}\right]+\frac{1}{2} \sigma_{Z_{j}}^{2}} Z_{j}\right)
\end{aligned}
$$

In general, we have that $\operatorname{Var}\left[V_{n+m}^{l}\right]<\operatorname{Var}\left[V_{n+m}\right]$ holds, unless $V_{n+m}^{l} \stackrel{d}{=} V_{n+m}$. Intuitively, it seems reasonable to choose $\Lambda$ such that $\operatorname{Var}\left[V_{n+m}^{l}\right]$ is maximized and hence as close as possible to $\operatorname{Var}\left[V_{n+m}\right]$.

In order to find an easy computable approximation, we propose to choose $\Lambda$ such that the first order approximation (31) for $\operatorname{Var}\left[V_{n+m}^{l}\right]$ is maximized:

$$
\Lambda=\sum_{j=0}^{n+m} \alpha_{j} e^{\mathrm{E}\left[Z_{j}\right]+\frac{1}{2} \sigma_{Z_{j}}^{2}} Z_{j}
$$

This means that the coefficients $\beta_{j}, j=1, \cdots, n+m$, in (10) are given by

$$
\beta_{j}=\sum_{i=0}^{j-1} \gamma_{i}=\sum_{i=0}^{j-1} \alpha_{i} e^{E\left[Z_{i}\right]+\frac{1}{2} \sigma_{Z_{i}}^{2}}=\sum_{i=0}^{j-1} \alpha_{i} e^{(n+m-i) \mu}
$$

Our main result is stated in the following Theorem.

Theorem 2 If the $\beta_{j}, j=1,2, \ldots, n+m$, are defined by (33), and if

$$
E\left[V_{n+m}\right]>0,
$$

then the quantiles of $W_{n+m}^{l}$ are given by

$$
Q_{p}^{+}\left[W_{n+m}^{l}\right]=\max [f(p), 0], \quad 0<p<1,
$$


whereas the d.f. of $W_{n+m}^{l}$ follows from

$$
f\left(F_{W_{n+m}^{l}}(x)\right)=x, \quad x \geq 0
$$

with $f(p)$ defined by (18).

Proof. It is straightforward to verify that the condition (34) implies that all $\beta_{j}>0$, for $j=1,2, \ldots n+m$. The stated result then follows from (26) and (27).

It is clear that any reasonable 'first saving- later consuming' plan should fulfill the condition (34), which states that the average final surplus $\mathrm{E}\left[V_{n+m}\right]$ should be non-negative.

\section{The case of constant savings and consump- tions}

In the remainder of this paper, we will consider the special case that all saving amounts are equal: $\alpha_{0}=\alpha_{1}=\cdots=\alpha_{n-1}=\alpha$, and also that all withdrawals are equal: $\alpha_{n}=\alpha_{n+1}=\cdots=\alpha_{n+m}=-1$. In the sequel, we will always assume that the $\beta_{j}$ coefficients, $j=1, \cdots, n+m$, needed to define $\Lambda$, are given by (33).

The condition (34) can be rewritten as

$$
\alpha>\alpha^{*}=\frac{1-e^{-(m+1) \mu}}{e^{n \mu}-1} .
$$

When the condition (37) is fulfilled, we find from Theorem 3 and (18) that the approximated quantiles $Q_{p}^{+}\left[W_{n+m}^{l}\right]$ and the approximated probabilities $F_{W_{n+m}^{l}}(x)$ follow from (35) and (36) with $f(p) \equiv f_{\alpha}(p)$ given by

$f_{\alpha}(p)=\alpha \sum_{i=0}^{n-1} e^{(n+m-i) \mu} e^{-\frac{1}{2} r_{i}^{2} \sigma_{Z_{i}}^{2}+r_{i} \sigma_{Z_{i}} \Phi^{-1}(p)}-\sum_{i=n}^{n+m} e^{(n+m-i) \mu} e^{\left.\frac{1}{2} r_{i}^{2} \sigma_{Z_{i}}^{2}+r_{i} \sigma_{Z_{i}} \Phi^{-1}(p)\right)}$.

Let $W_{n+m}(\alpha)$ and $W_{n+m}^{l}(\alpha)$ denote the (approximated) final wealth for a given saving-consumption plan with a saving $\alpha$ and consumption level of 1 . Similarly, $V_{n+m}(\alpha)$ is the surplus of the $\alpha$ - plan. 
For $\alpha^{*}$ as defined in (37), one has that

$$
F_{W_{n+m}^{l}\left(\alpha^{*}\right)}(0)>0.5 .
$$

Indeed, $\alpha^{*}$ corresponds to the saving-consumption plan with expected surplus $\mathrm{E}\left[V_{n+m}\left(\alpha^{*}\right)\right]$ equal to zero. From (8), we find

$$
E\left[V_{n+m}\left(\alpha^{*}\right)\right]=a^{*} \sum_{i=0}^{n-1} e^{(n+m-i) \mu}-\sum_{i=n}^{n+m} e^{(n+m-i) \mu}=0 .
$$

From this expression we see that all $\beta_{j}, j=1, \cdots, n+m$, as defined in (33) are strictly positive. Hence, from (27) we find that the probability of consumption shortfall follows from

$$
f_{\alpha^{*}}\left(F_{W_{n+m}^{l}\left(\alpha^{*}\right)}(0)\right)=0
$$

From (18) and the fact that the sequence $\left\{r_{i} \sigma_{Z_{i}}\right\}_{0 \leq r \leq n+m}$ is non-increasing and positive, one finds that

$$
f_{\alpha^{*}}(0.5)=a^{*} \sum_{i=0}^{n-1} e^{(n+m-i) \mu} e^{-\frac{1}{2} r_{i}^{2} \sigma_{Z_{i}}^{2}}-\sum_{i=n}^{n+m-1} e^{(n+m-i) \mu} e^{-\frac{1}{2} r_{i}^{2} \sigma_{Z_{i}}^{2}}<0 .
$$

so that $F_{W_{n+m}^{l}\left(\alpha^{*}\right)}(0)>0.5$, as stated in (39).

In practical situations, one will often be interested in the minimal savings amount $\alpha$ that is required such that the probability of a consumption shortfall $F_{W_{n+m}(\alpha)}(0)$ is at most equal to $\varepsilon$. Hence, let us consider the case that one wants to determine $\alpha(\varepsilon)$ which is determined by

$$
\alpha(\varepsilon)=\inf \left\{\alpha \mid F_{W_{n+m}(\alpha)}(0) \leq \varepsilon\right\}, \quad 0<\varepsilon<1 .
$$

The probability $\varepsilon$ is typically smaller than $10 \%$, let's say.

In general, $F_{W_{n+m}(\alpha)}(0)$ is strictly decreasing and continuous in $\alpha$. This implies that $\alpha(\varepsilon)$ follows from

$$
F_{W_{n+m}(\alpha(\varepsilon))}(0)=\varepsilon .
$$

We propose to approximate $\alpha(\varepsilon)$ by $\alpha^{l}(\varepsilon)$ which is defined by

$$
F_{W_{n+m}^{l}\left(\alpha^{l}(\varepsilon)\right)}(0)=\varepsilon
$$


Now, in case $\varepsilon$ is such that $\alpha^{l}(\varepsilon)>\alpha^{*}$ we have that $E\left[V_{n+m}\left(\alpha^{l}(\varepsilon)\right)\right]>0$. Hence, from (36), we have that the approximated savings effort $\alpha^{l}(\varepsilon)$ can be found from

$$
f_{\alpha^{l}(\varepsilon)}(\varepsilon)=0 \quad \text { if } \alpha^{l}(\varepsilon)>\alpha^{*} .
$$

Notice that we can expect that for any reasonable $\varepsilon<0.5$, the approximated savings effort $\alpha^{l}(\varepsilon)$ can be found from (44). To see this, notice that from (39) we have that $F_{W_{n+m}^{l}\left(\alpha^{*}\right)}(0)>0.5$. Furthermore, we have that $F_{W_{n+m}(\alpha)}(0)$ is in general strictly decreasing and continuous in $\alpha$. From these two observations we find that it is very likely that for a typical $\varepsilon<0.5$ we will have that the condition $\alpha^{l}(\varepsilon)>\alpha^{*}$ in (44) is fulfilled.

\section{Numerical illustration}

In this section we will numerically illustrate the results of this paper, applied to the special case of $\alpha$-plans as considered in the previous section. We will assume that the yearly returns $Y_{i}$ have expectation and variance given by $\mu$ $-\frac{\sigma^{2}}{2}$ and $\sigma^{2}$, where $\mu=0.075$ and $\sigma=0.15$.

First, we consider a saving-consumption plan that consists of 10 constant savings $\alpha_{0}=\alpha_{2}=\cdots=\alpha_{9}=1$, followed by 10 constant withdrawals $\alpha_{10}=\alpha_{11}=\cdots=\alpha_{19}=-1$. In this case we find that $\mathrm{E}\left[V_{19}\right]=16.02>0$, so that Theorem 3 can be used for determining the quantiles of $W_{19}^{l}$.

In Table 1 we compare the approximated quantiles $Q_{p}^{+}\left[W_{19}^{l}\right]$ with the simulated quantiles ' $Q_{p}^{+}\left[W_{19}\right]$ ' This table illustrates the accuracy of the lower bound based approximation $W_{19}^{l}$. From this table we find for instance that there is a $90 \%$ probability that the final wealth at time 19 will exceed 1.75 (simulated value). The approximated value for this final wealth is 1.76.

From (27) it follows that the (approximated) probability of consumption shortfall $\operatorname{Pr}\left[W_{19}^{l}=0\right]$ equals $4.83 \%$. In case the investor finds this probability too high, he will have to increase his savings efforts of 1 per year during the first ten years.

As a second application, we consider the 20/65/95 saving-consumption plan as mentioned in the introduction. This plan consists of 45 constant savings $\alpha_{0}=\alpha_{2}=\cdots=\alpha_{44}=\alpha>0$, the first one at the age of 20 and the last one at the age of 64 . After retirement, yearly withdrawals equal to -1 will be made until the age of 95 has been reached, hence $\alpha_{45}=\alpha_{46}=\cdots=$ $\alpha_{75}=-1$.

The condition (34), or equivalently (37) can be expressed as: $\alpha>0.031966$. 


\begin{tabular}{|l||l|l|l|}
\hline$p$ & $Q_{p}^{+}\left[W_{19}^{l}\right]$ & $' Q_{p}^{+}\left[W_{19}\right]$ & s.e. \\
\hline \hline 0.95 & 45.11 & 45.17 & 0.28 \\
0.90 & 34.81 & 34.80 & 0.16 \\
0.75 & 21.88 & 21.89 & 0.10 \\
0.50 & 12.11 & 12.13 & 0.01 \\
0.25 & 5.64 & 5.68 & 0.03 \\
0.10 & 1.76 & 1.75 & 0.04 \\
0.01 & 0 & 0 & 0.00 \\
\hline
\end{tabular}

Table 1: Approximate and simulated values for the quantiles of $W_{19}$.

\begin{tabular}{|c||c|}
\hline$\alpha$ & $\operatorname{Pr}\left[W_{75}^{l}=0\right]$ \\
\hline \hline 0.0320 & $71.29 \%$ \\
0.0500 & $55.38 \%$ \\
0.1000 & $23.22 \%$ \\
0.1500 & $9.89 \%$ \\
0.2500 & $2.24 \%$ \\
0.5000 & $0.14 \%$ \\
\hline
\end{tabular}

Table 2: The approximated probability of consumption shortfall for different values of the savings effort.

Table 2 contains the approximated probabilities of consumption shortfall $\operatorname{Pr}\left[W_{75}^{l}=0\right]$, for different saving amounts $\alpha$. These probabilities follow from (27).

From (44) we find that the approximated minimal savings amount $\alpha^{l}(0.05)$ that guarantees that the probability of consumption shortfall is less than or equal to $5 \%$ is given by 0.1935 .

Acknowledgement 3 The authors acknowledge the financial support by the Onderzoeksfonds K.U.Leuven (GOA/02: Actuariële, financiële en statistische aspecten van afhankelijkheden in verzekerings- en financiële portefeuilles). 


\section{References}

[1] Albrecher, H., Dhaene, J., Goovaerts, M.J. \& Schoutens, W. (2003). "Static hedging of Asian options under Lévy models: the comonotonic approach", Research Report OR 0365, Department of Applied Economics, K.U.Leuven.

[2] Dhaene, J., Denuit, M., Goovaerts, M.J., Kaas, R. \& Vyncke, D. 2002(a). "The concept of comonotonicity in actuarial science and finance: Theory", Insurance: Mathematics \& Economics, 31(1), 3-33.

[3] Dhaene, J., Denuit, M., Goovaerts, M.J., Kaas, R. \& Vyncke, D., 2002(b). "The concept of comonotonicity in actuarial science and finance: Applications", Insurance: Mathematics \& Economics, 31(2), 133-161.

[4] Dhaene, J., Vanduffel, S., Goovaerts, M.J., Kaas, R. \& Vyncke, D. (2004). "Comonotonic approximations for optimal portfolio selection problems", Journal of Risk and Insurance, to appear

[5] Dhaene, J., Vanduffel, S., Tang, Q., Goovaerts, M.J., Kaas, R. \& Vyncke, D. (2004). "Solvency capital, risk measures and comonotonicity: a review", Research Report OR 0416, Department of Applied Economics, K.U.Leuven.

[6] Goovaerts, M.J., De Schepper, A., Hua, Y., Darkiewicz, G. \& Vyncke, D. (2003). "An investigation on the use of copulas when calculation general cash flow distributions", Proceedings of the 8th International Congress on Insurance: Mathematics and Economics, Rome, June, 14-16.

[7] Kaas, R., Dhaene, J. \& Goovaerts, M. (2000). "Upper and lower bounds for sums of random variables", Insurance: Mathematics and Economics, $27,151-168$.

[8] Milevsky, M.A. \& Posner, S.E. (1998). "Asian Options, the Sum of Lognormals, and the Reciprocal Gamma Distribution", Journal of financial and quantitative analysis, 33(3), 409-422.

[9] Milevsky, M.A. \& Robinson C. (2000). "Self-Annuitization and Ruin in Retirement", North American Actuarial Journal, 4(4), 112-124. 
[10] Vanduffel, S., Dhaene, J., Goovaerts, M.J. \& Kaas, R. (2003). "The hurdle-race problem", Insurance: Mathematics \& Economics, 33(2), 405-413.

[11] Vanduffel, S., Hoedemakers, T. \& Dhaene, J. (2004). "Comparing approximations for sums of non-independent lognormal random variables", Research Report OR 0418, Department of Applied Economics, K.U.Leuven.

[12] Vanmaele, M., Dhaene, J., Deelstra, G., Liinev, J., Goovaerts M.J. (2004). "Bounds for the price of discretely sampled arithmetic Asian options", European Journal of Operational Research, to appear. 


